hep-ph/9812271

SMU-HEP/98-08

\title{
Asymptotic high energy behavior of the deeply virtual Compton scattering amplitude市
}

\author{
B.I. Ermolaev \\ A.F. Ioffe Physico-Technical Institute, St. Petersburg, 194021, \\ Russia \\ F. Olness \\ Southern Methodist University, Dallas, TX 75275, USA \\ A.G. Shuvaev \\ St. Petersburg Nuclear Physics Institute, Gatchina, \\ St. Petersburg, 188350, Russia
}

\begin{abstract}
We compute the deeply virtual Compton scattering (DVCS) amplitude for forward and backward scattering in the asymptotic limit. Since this calculation does not assume ordering of the transverse momenta, it includes important logarithmic contributions that are beyond those summed by the DGLAP evolution. These contributions lead to a power-like behavior for the forward DVCS amplitude.
\end{abstract}

\footnotetext{
${ }^{\dagger}$ This work is supported in part by Grant INTAS-RFBR-95-311, Grant RFBR-98-02-
} 17629, the US Department of Energy, and the Lightner-Sams Foundation. 


\section{Introduction}

The deeply virtual Compton scattering (DVCS) has received attention recently since it provides a new and interesting theoretical framework for perturbative and nonperturbative QCD applications. 11, 2, 3] The basic mechanism of this process is reminiscent of the usual deeply inelastic (DIS) leptonhadron scattering process which begins with the absorption of a virtual photon by the quark constitutient. The DVCS differs from DIS in the second step when the same quark constitutient then emits an on-shell photon. The kinematics of the DVCS reaction are characterized by a high invariant energy and a large virtuality of the initial photon compared to the quark mass.

The DVCS amplitude is, in a sense, a generalization of the conventional deep inelastic structure (DIS) functions, and can be expressed using asymmetric distribution functions. To incorporate the radiative corrections into the DVCS amplitude, the conventional DGLAP evolution equations [4] have been used [1, 2, 3] (for review on the DGLAP evolution for the DVCS see [5] and references therein). As a typical for the DGLAP result, the exponential asymptotical high energy behavior of the DVCS amplitude was obtained. Such asymptotics are true for the kinematical region where the invariant total energy and the virtuality of the off-shell photon are of the same order, i.e. in so-called the "hard" kinematics. However, in order to obtain the DVCS asymptotics in the other kinematical regions, and in particular in the "semihard" (or the Regge type) one, with the invariant total energy being much greater than the off-shell photon virtuality, a generalized set of evolution equations is required.

Although predictions based on the DGLAP evolution yield good agreement with present experimental deep inelastic scattering data, 6, 7, 8] from the theoretical point of view this approach is insufficient when the kinematics are of the Regge type where the total invariant energy is much greater than the other invariants, including the virtuality of the deeply virtual photon. In this kinematic region, the DGLAP equations can not account for contributions independent of this virtuality. Taking these contributions into account for the DIS structure functions led to a power-like dependence on the total energy both for photon scattering from an unpolarized quark (the BFKL Pomeron [9]) and for the scattering from a polarized quark. 10]

Naturally, one can expect a similar change in the asymptotic behavior of the DVCS amplitude in the Regge limit. To demonstrate this behavior, let us 


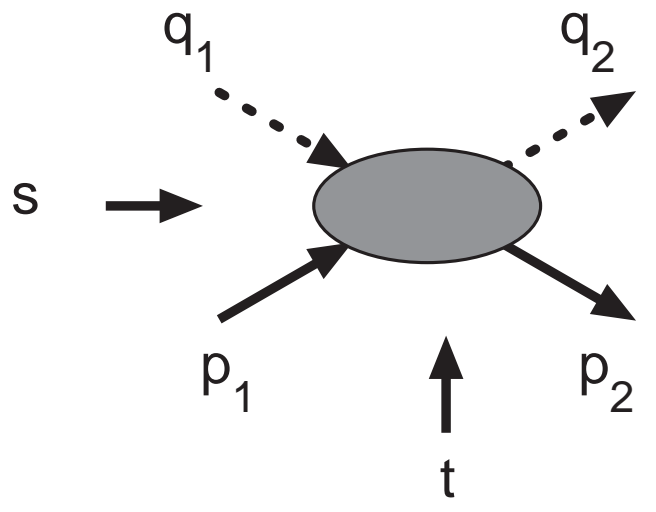

Figure 1: The fundamental DVCS process: scattering of a deeply virtual photon $\left(q_{1}\right)$ off a nearly on-shell light quark $\left(p_{1}\right)$ to produce an on-shell photon $\left(q_{2}\right)$ and a quark $\left(p_{2}\right)$.

consider the scattering of a deeply virtual photon off a nearly on-shell light quark which produces a photon and a quark-both on-shell, (cf., Fig.1). In Fig.1, $s, t$ and $u$ are the standard Mandelstam variables:

$$
\begin{aligned}
s & =\left(p_{1}+q_{1}\right)^{2} \\
t & =\left(q_{2}-q_{1}\right)^{2} \\
u & =\left(q_{2}-p_{1}\right)^{2} .
\end{aligned}
$$

In the Born approximation, only the two Feynman graphs in Fig.2 contribute to the DVCS process: Beyond the Born approximation the value of the QCD-radiative corrections strongly depend on the kinematics of the scattering. In particular, in the two regions

$$
\begin{array}{ll}
s \sim-u \gg-t & \text { Forward Region } \\
s \sim-t \gg-u & \text { Backward Region }
\end{array}
$$

the QCD radiative corrections are large, whereas in the region $s \sim-t \sim-u$ they are comparatively small. In the center of mass system (CMS), $s \sim$ 

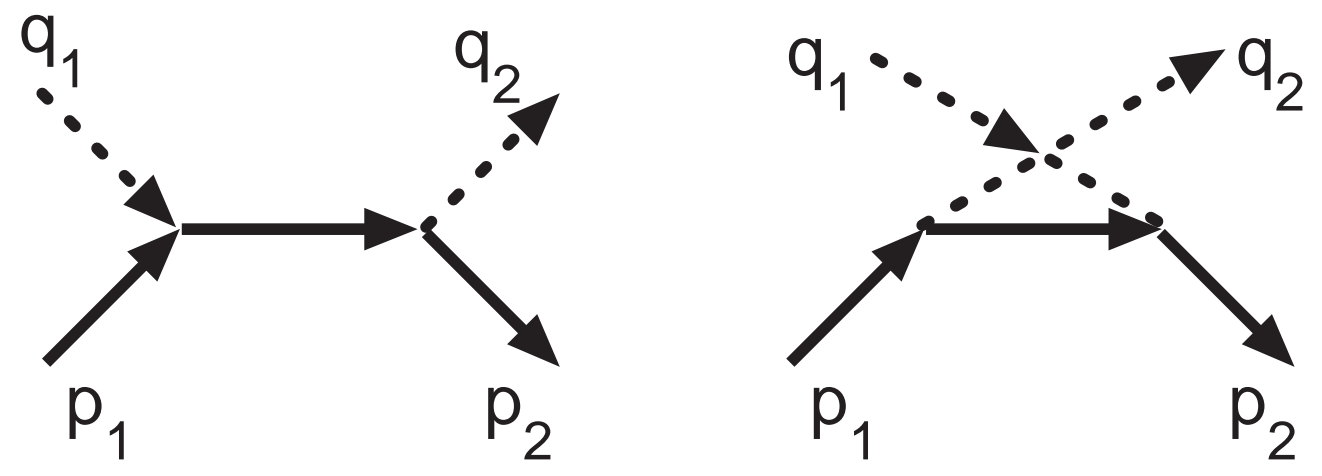

Figure 2: Born graphs for the DVCS process.

$-u \gg-t$ corresponds to the Forward Region (Eq. (2)) (small scattering angles), and $s \sim-t \gg-u$ corresponds to the Backward Region (Eq. (3)).

Both of the above regions are of the Regge type. When

$$
s \gg Q^{2},
$$

where $Q^{2}=-q_{1}^{2}$ is the photon virtuality, the Regge theory predicts a powerlike $s$-dependence for the DVCS amplitude in each of the regions. Such behavior is result of summing the QCD-radiative corrections to all orders in the QCD-coupling.

Below we obtain the asymptotical behavior of the DVCS amplitudes for the "semi-hard" kinematical regions (Eq. (2),Eq. (田); Eq. (3),Eq. (四)) in the leading logarithmic approximation (LLA) by solving the Infrared Evolution Equations (IREE) [11, 12] for them. As in the case of the DGLAP evolution, in order to obtain the evolution in the Regge regions above, we consider the DVCS from an on-shell quark. Recently, the IREE method has been used to obtain the small- $x$ asymptotic limit of the DIS spin-dependent structure functions [10]. While references [1, 2, 3] include consideration of the hadronic component, we study only the partonic part of the DVCS which can be investigated with the perturbative methods of QCD. Since we consider the DVCS from a quark, our results depend on the factorization scale $\mu$; this 
factorization scale dependence will cancel out in the expression for the DVCS from a hadron. Operationally, within the LLA-accuracy, this effects the replacement of $\mu$ by some mass factor of order of the hadron mass.

The paper is organized as follows: In Sect. 2 we briefly review the IREE method we shall use to obtain the expression for the DVCS amplitude in the forward region, Eq. (21), Eq. (4). In Sect. 3 we investigate the DVCS amplitude in the backward region, Eq. (3), Eq. (田). In Sect. 4 we calculate the non-forward structure functions for DVCS in the Regge region Eq. (4), and express them in terms of the Reggeons corresponding to on-shell scattering. Finally, in Sect. 5 we discuss the implications of these results.

\section{Forward DVCS}

Our calculation will follow the general prescription of the IREE method given in Ref. [11, 12]. All the virtual momenta, $k_{i}$, can be expressed through the Sudakov parameterization as follows:

$$
\begin{aligned}
k_{i} & =\alpha_{i} p_{1}+\beta_{i} q_{1}^{\prime}+k_{\perp}, \\
q_{1}^{\prime} & =q_{1}-\frac{q_{1}^{2}}{2 p_{1} \cdot q_{1}} p_{1} \\
k_{\perp} \cdot p_{1} & =k_{\perp} \cdot q_{1}=0
\end{aligned}
$$

where we have neglected the masses of incoming and outgoing quarks.

In order to avoid infrared singularities, it is necessary to introduce an infrared cut-off $\mu$ in transverse momentum space so that

$$
k_{i}^{\perp}>\mu \text {. }
$$

In the LLA, such regularization does not violate gauge invariance; therefore the Gribov bremsstrahlung theorem[13] is applicable in this case. ( $C f$., Ref. [14, 15].) The essence of this theorem is that the virtual gluon with the minimal $k_{\perp}$ can be factorized out of the amplitude. Consequently, the principle contribution comes from graphs where the gluon propagator is attached

only to external lines. Additionally, the integrations over the remaining gluons do not depend on the longitudinal momentum of this factorized gluon. There is a dependence on the factorized gluon's transverse momentum - this $k_{\perp}$ becomes a new infrared cut-off for the remaining integrations. 
Since QCD-radiative corrections in the LLA do not violate the spin structure of the Born approximation, the forward DVCS amplitude, $M_{F}$, is given by:

$M_{F}=M_{F}^{\text {Born }} \Phi\left[\ln \left(s / \mu^{2}\right), \ln \left(Q^{2} / \mu^{2}\right), \ln \left(-t / \mu^{2}\right)\right] \equiv M_{F}^{\text {Born }} \Phi\left[z_{1}, z_{2}, z_{3}\right]$

where we define

$$
\begin{aligned}
& z_{1}=\ln \left(s / \mu^{2}\right), \\
& z_{2}=\ln \left(Q^{2} / \mu^{2}\right) \\
& z_{3}=\ln \left(-t / \mu^{2}\right)
\end{aligned},
$$

Although the value of $\mu^{2}$ is not fixed, we presume it is much less than any of $s, Q^{2}$, or $-t$. Instead of setting $\mu^{2}$ to a fixed value, let us differentiate $M_{F}$ with respect to $\mu$ to obtain our IREE:

$$
-\mu^{2} \frac{\partial M_{F}}{\partial \mu^{2}}=\frac{\partial M_{F}}{\partial z_{1}}+\frac{\partial M_{F}}{\partial z_{2}}+\frac{\partial M_{F}}{\partial z_{3}} .
$$

The LHS of Eq. (11) provides half of the IREE we desire for $M_{F}$, (cf., Fig.3). In order to obtain the remaining half of the IREE, we should look for the virtual quark or a gluon with the minimal transverse momentum.

If the particle with minimal transverse momentum is a gluon, then it can be factorized, (graph (b) in RHS of Fig.3). Integration over the longitudinal momentum of the gluon yields (after differentiation over $\ln \left(\mu^{2}\right)$ ),

$$
-2 \lambda z_{3} M_{F}
$$

with

$$
\lambda=\alpha_{s} C_{F} / 4 \pi
$$

$C_{F}=\left(N^{2}-1\right) / 2 N$, and $N=3$.

If the particle with minimal transverse momentum is a quark, then the process is more involved. In order to evaluate the leading-log (LL) contribution, there should be another quark on the ladder with minimal $k_{\perp}$ from the same ladder rung (graph (c) in RHS of Fig.3). However, as long as we keep $-t \gg \mu^{2}$, the LL contribution arises only when $k_{\perp}^{2}>-t$. Then the integration region over $k_{\perp}$ does not include $\mu$ as the lower limit when $-t \gg \mu^{2}$, and 

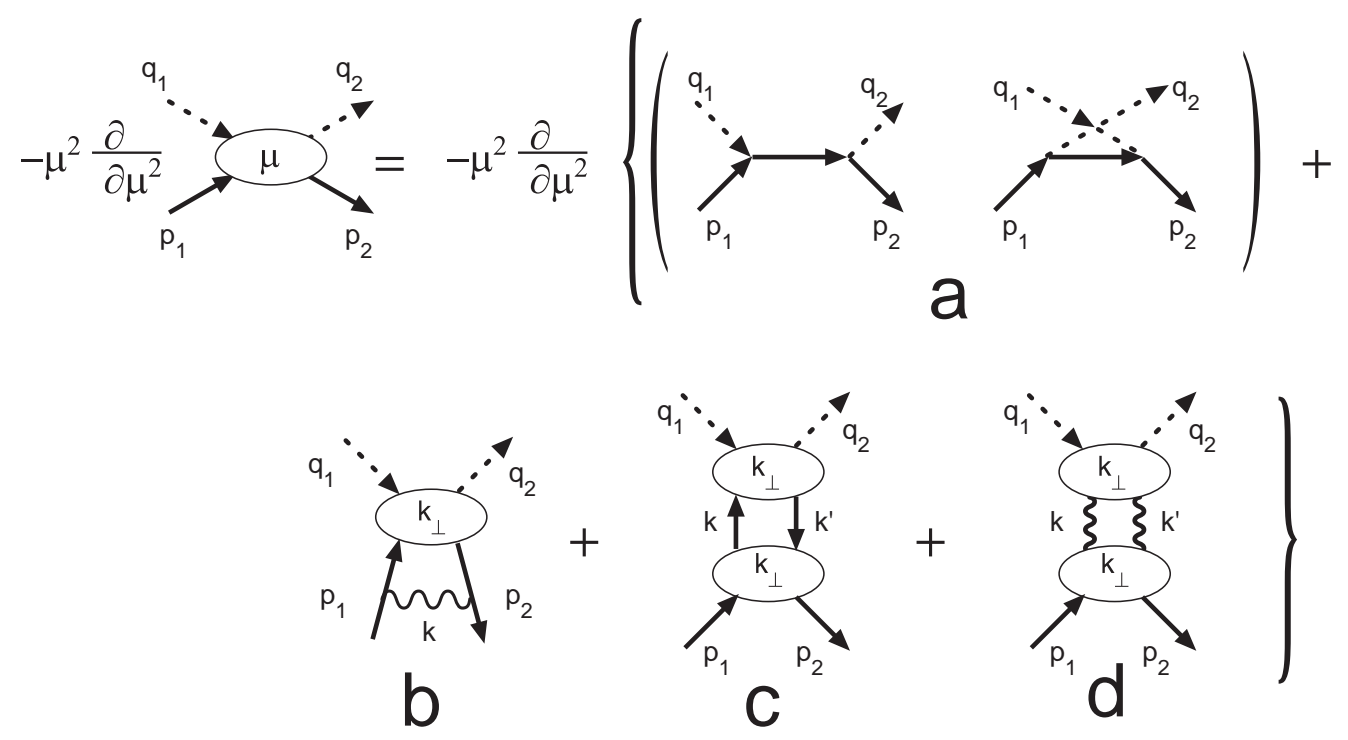

Figure 3: The general form of the evolution equations for DVCS.

the whole term (c) in Fig.3 will be independent of $\mu$, and any derivatives w.r.t. $\mu$ will vanish.

By the same reasoning, we can dismiss the case where, instead of quarks, two ladder gluons have minimal values of $k_{\perp}$, (corresponding to graph (d) in Fig.3).

The Born contribution (graph (a) in Fig.3) also does not depend on $\mu$ when $-t \gg \mu^{2}$, and this term also vanishes when differentiated w.r.t. $\mu$.

We therefore find the RHS of the IREE is given solely by graph (b) in Fig.3, and we obtain

$$
\frac{\partial M_{F}}{\partial z_{1}}+\frac{\partial M_{F}}{\partial z_{2}}+\frac{\partial M_{F}}{\partial z_{3}}=-2 \lambda z_{3} M_{F}
$$

The solution of this IREE is:

$$
M_{F}=\phi\left(z_{1}-z_{3}, z_{2}-z_{3}\right) e^{-\lambda z_{3}^{2}}
$$


with $\phi$ to be defined below in Eq. (17).

We will discuss now the kinematic region where

$$
s>Q^{2}>-t
$$

We then have $-t=\mu^{2}$, which implies $z_{3}=\ln \left(-t / \mu^{2}\right)=0$, and consequently $\phi\left(z_{1}-z_{3}, z_{2}-z_{3}\right) \rightarrow \phi\left(z_{1}, z_{2}\right)$. In this kinematic limit we define

$$
\widetilde{M_{F}}=\phi\left(z_{1}, z_{2}\right)
$$

where $\widetilde{M_{F}}$ is the DVCS amplitude for the scattering at (nearly) zero angle, i.e. for $-t=\mu^{2}$. We define the function $\phi$ using the matching condition of Eq. (17); therefore, we require an independent calculation of $\widetilde{M_{F}}$ to obtain $\phi$. This amplitude does not depend on $z_{3}$. Thus, we have re-expressed the scattering amplitude in terms of a simpler set of variables, and eliminated one variable (namely, the smallest one). Let us note that when $t \neq 0$, the DVCS, even when regarded as the elastic $2 \rightarrow 2$ - process, is always accompanied by bremsstrahlung consisting of photons and gluons with energies less than $\sqrt{-t}$. A part of them have energies less than $\mu$. Such very soft radiation does not affect kinematics of the DVCS. In the LLA it would factorize out of the scattering amplitude (for details see [14]), and would cancel with virtual gluon/photon contributions of the same energy scale in expressions for the cross sections. Through the present paper we neglect both the emission and the radiative corrections for bremsstrahlung with energies below $\mu$. So, within LLA-accuracy we identify $\tilde{M}_{F}$ as the usual DVCS-amplitude with $t=0$. While we begin our calculation with a complicated process involving off-shell exchanges, our final result involves elastic scattering amplitudes with only on-shell particles. For the DVCS process, the relevant process is the elastic quark-photon scattering amplitude with all the particles on-shell.

We now construct the IREE for $\widetilde{M_{F}}$ as defined in Eq. (17). Recall that we are working in the limit where $z_{3}=\ln \left(-t / \mu^{2}\right)=0$. In the following, we will show that the only contributions to $\widehat{M_{F}}$ come from graphs (a), (c), (d) of Fig.3. Let us now demonstrate this result. When the incoming and the outgoing quarks in Fig.1 are polarized, there are spin-dependent, as well as spin-independent, contributions to the DVCS amplitude. In the Born approximation, the spin-independent contribution is symmetric with respect to permutations of $s$ and $u$, whereas the spin-dependent contribution is antisymmetric. This is also true when radiative corrections in the LLA are taken 
into account. Therefore, the spin-independent part of the DVCS amplitude has a positive signature, the spin-dependent part has a negative signature.

Consequently, we find it convenient to decompose the DVCS amplitude into a spin-dependent amplitude $N_{F}$, and a spin-independent amplitude $U_{F}$ :

$$
M_{F}=N_{F}+U_{F}
$$

We make the same decomposition for the $\widetilde{M_{F}}$, the DVCS amplitude in the $z_{3}=0$ limit:

$$
\widetilde{M_{F}}=\widetilde{N_{F}}+\widetilde{U_{F}}
$$

The $t$-dependence of $M_{F}$ is given by Eq. (15) provided $\widetilde{M_{F}}$ is known.

Let us first discuss the spin-dependent part, $\widetilde{N_{F}}$, of the DVCS amplitude $\widetilde{M_{F}}$. If we neglect mass of the initial/final state quark, the quark spin is then collinear to its momentum. Since the transverse spin contribution is proportional to the quark mass, it is negligible in this limit.

For simplicity, we consider the case where the QCD-radiative corrections to the Born approximation correspond to adding extra gluon propagators to both graphs of Fig.2. In this case, term (d) of Fig.3 (with a two-gluon intermediate state) does not contribute to to the RHS of IREE. Neither terms (a) and (b) also do not contribute because $t=0$.

We implicitly define $\widetilde{F}$ via a Mellin transform

$$
\widetilde{N}_{F}\left(z_{1}, z_{2}\right)=\int_{-\imath \infty}^{\imath \infty} \frac{d \omega}{2 \pi \imath} e^{z_{1} \omega} \xi(\omega) \widetilde{F}\left(\omega, z_{2}\right)
$$

with the negative signature factor

$$
\xi(\omega)=\frac{e^{-\imath \pi \omega}-1}{2} \approx \frac{-\imath \pi \omega}{2},
$$

The IREE for the spin-dependent part takes the following form:

$$
\left(\frac{\partial}{\partial z_{2}}+\omega\right) \widetilde{F}\left(\omega, z_{2}\right)=\frac{1}{8 \pi^{2}} \widetilde{F}\left(\omega, z_{2}\right) f_{0}(\omega)
$$

where $f_{0}$ is the Mellin amplitude for quark-quark elastic scattering with all quarks on-shell. It corresponds to the lowest blob of graph (c) in Fig.3., and explicit expression was obtained in Ref. [11]:

$$
f_{0}=4 \pi^{2}\left\{\omega-\left[\omega^{2}-\frac{g^{2} C_{F}}{2 \pi^{2}}\left(1-\frac{1}{2 \pi^{2} \omega} \psi(\omega)\right)\right]^{1 / 2}\right\},
$$


with

$$
\psi(\omega)=g^{2} N \frac{d}{d y} \ln \left(e^{y^{2} / 4} D_{\frac{-1}{2 N^{2}}}(y)\right),
$$

and

$$
y=\omega / \sqrt{g^{2} N / 8 \pi^{2}},
$$

where $g=\sqrt{4 \pi \alpha_{s}}$ is the QCD-coupling (which is fixed in the LLA), and $D_{\frac{-1}{2 N^{2}}}$ is the parabolic cylinder (or Webber) function.

The solution of Eq. (22) is

$$
\widetilde{F}\left(\omega, z_{2}\right)=\widetilde{\phi}(\omega) e^{-\left(\omega-f_{0} / 8 \pi^{2}\right) z_{2}} .
$$

Thus,

$$
\widetilde{N_{F}}\left(z_{1}, z_{2}\right)=\int_{-\imath \infty}^{\imath \infty} \frac{d \omega}{2 \pi \imath} \phi(\omega) \xi(\omega)\left(\frac{s}{Q^{2}}\right)^{\omega}\left(\frac{Q^{2}}{\mu^{2}}\right)^{f_{0}(\omega) / 8 \pi^{2}}
$$

Eq. (27) fixes the anomalous dimension

$$
\gamma \equiv f_{0}(\omega) / 8 \pi^{2}
$$

for $\widetilde{N_{F}}$. The unknown function $\widetilde{\phi}(\omega)$ can be fixed by the matching condition:

$$
\widetilde{N_{F}}=C\left(z_{1}\right)
$$

when $z_{2}=0$. The new amplitude $C\left(z_{1}\right)$ in Eq. (27) is the amplitude of the elastic Compton scattering with both photons on-shell. The IREE for $C\left(z_{1}\right)$ is obtained by canceling the $z_{2}$-dependence in the LHS, and adding to RHS the contribution of term (a) in Fig. 3 which now is $\mu$-dependent. Expressions for the anomalous dimensions of the spin-dependent structure function $g_{1}$ incorporating all nonleading order contributions were obtained in Ref. 12 in the double logarithmic approximation. The resummation of the $\mathcal{O}\left(\alpha_{s}^{n+1} \ln ^{2 n} x\right)$ term in the singlet evolution equation was performed in Ref. [7].

Combining the above formulas Eq. (15), Eq. (17), Eq. (18)-Eq. (25), we arrive at the result for the spin-dependent part of the DVCS amplitude:

$$
N_{F}=\frac{e^{2}}{g^{2} C_{F}} \int_{-\imath \infty}^{\imath \infty} \frac{d \omega}{2 \pi \imath}\left(\frac{s}{Q^{2}}\right)^{\omega} \frac{1}{\omega-f_{0} / 8 \pi^{2}}\left(\frac{Q^{2}}{\mu^{2}}\right)^{f_{0} / 8 \pi^{2}} e^{-\frac{\alpha_{s} C_{F}}{4 \pi} \ln ^{2}\left(-t / \mu^{2}\right)}
$$


where $e^{2}=\sqrt{4 \pi \alpha}$. To identify the contribution to the spin-dependent DVCS amplitude from the Born process (term (a) in Fig.3), we neglect the difference between the quark mass and $\mu$ :

$$
N_{F}^{\text {Born }}=\int_{-\imath \infty}^{\imath \infty} \frac{d \omega}{2 \pi i}\left(\frac{s}{\mu^{2}}\right)^{\omega} \frac{e^{2}}{\omega} \xi(\omega)
$$

The asymptotic high energy behavior in the forward region, Eq. (2), for $N_{F}$ is (cf., Ref. [10]):

$$
N_{F} \simeq\left(\frac{s}{Q^{2}}\right)^{a}\left(\frac{Q^{2}}{\mu^{2}}\right)^{a / 2} e^{-\frac{\alpha_{s}}{4 \pi} \ln ^{2}\left(-t / \mu^{2}\right)}
$$

with

$$
a=\sqrt{\frac{2 \alpha_{s} C_{F}}{\pi}\left(1+\frac{1}{2 N^{2}}\right)} .
$$

We can incorporate term (d) of Fig.3 into the IREE for $F_{N}$ as was done in in Ref. [10. The result is to change $a$ in Eq. (33) to

$$
a \simeq 3.5 \sqrt{\frac{2 \alpha_{s}}{\pi} \frac{N}{4}}
$$

We now discuss $\widetilde{U_{F}}$, the spin-independent part of $\widetilde{M_{F}}$. Thus, we are interested in the portion of the amplitude with the positive signature. For $t=0$, term (b) in Fig.3 does not contribute. In contrast to the spin-dependent part of the DVCS amplitude, the contribution of term (c) is now small compared to term (d) by a power of $s$; therefore we neglect term (c). The negligible contribution of term (c) can be anticipated since the Regge theory predicts the asymptotic behavior of the scattering amplitude to be $\sim s^{\left(j_{1}+j_{2}+. .\right)-n+1}$, where $n$ is the number of $t$-channel intermediate particles exchanged, and $j_{i}$ are their spins.

Repeating the spin-independent calculation as in the previous spin-dependent case, we obtain the asymptotic behavior of $U_{F}$ in the Regge limit of the forward region, Eq. (2):

$$
U_{F} \sim R\left(s, Q^{2}\right) e^{-\frac{\alpha_{s} N}{4 \pi} \ln ^{2}\left(-t / \mu^{2}\right)}
$$


where $R\left(s, Q^{2}\right)$ is the amplitude for Compton scattering. Since this process is mediated by the Pomeron, we obtain

$$
R\left(s, Q^{2}\right) \sim\left(\frac{s}{Q^{2}}\right)^{1+\Delta_{P}}\left(\frac{Q^{2}}{\mu_{2}}\right)^{\gamma_{P}},
$$

where $\Delta_{P}$ is the Pomeron intercept, and $\gamma_{P}$ is the Pomeron anomalous dimension.

Writing the virtual photon momentum as $q_{1}=q^{\prime}-\zeta p$ DVCS amplitude can be expressed in terms of the asymmetry parameter $\zeta, Q^{2}=\zeta s$.

\section{Backward DVCS}

The backward DVCS process is straightforward to analyze given the previous forward DVCS calculation. Since, in the Born approximation, the contribution of graph (b) is much greater than graph (a), we can neglect graph (a). Furthermore, neither graph (c) nor graph (d) contributes to the IREE in the backward region.

Recall that the kinematic configuration in the backward region is specified by (Eq. (3)):

$$
s \sim-t \gg-u \quad \text { Backward Region }
$$

Therefore, intermediate partons with minimal value of $k_{\perp}$ can only exist in the $u$-channel (when $u \simeq 0$ ). The $u$-channel two particle intermediate state can not consist of two quarks or two gluons due to fermion charge conservation. Instead, a new contribution given by graph in Fig.4 can now contribute. However, it is known that this contribution is beyond the LLA. Therefore, only terms (a) and (b) of Fig.3 contribute to the RHS of the IREE in the backward region.

Finally, we note that there is no difference between asymptotic behavior of the polarized and unpolarized parts of the backward DVCS amplitude $M_{B}$. $M_{B}$ depends on the variables $z_{1}, z_{2}$, and a new variable $z_{4}$ defined as:

$$
z_{4}=\ln \left(-u / \mu^{2}\right)
$$

For backward scattering $z_{1} \approx z_{3}$, so these variables are not independent. In a similar manner, there was no $z_{4}$-dependence for the forward DVCS amplitude 


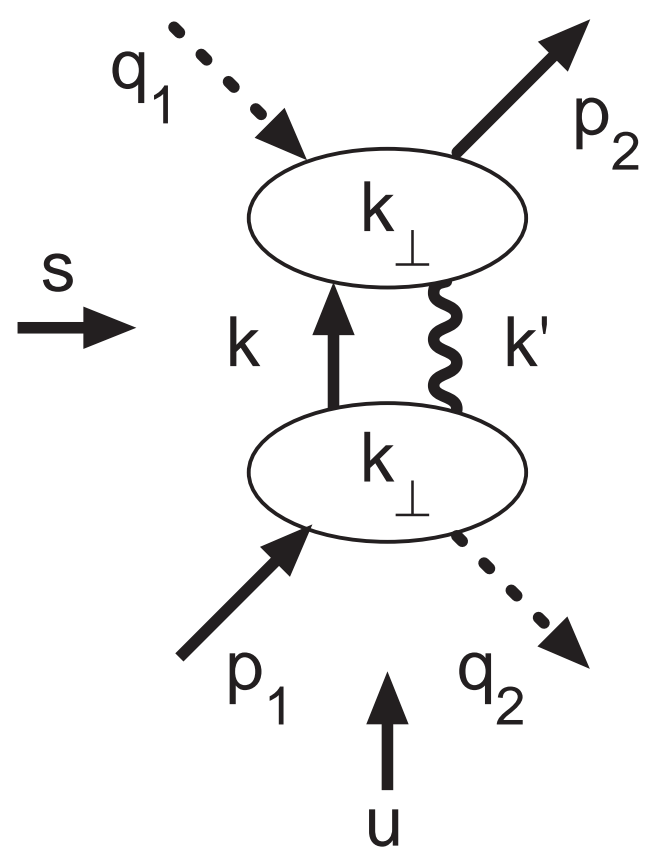

Figure 4: The two-particle intermediate state for the backward DVCS process.

because $z_{4} \approx z_{1}$ in this region. The IREE for $M_{B}$ is (cf., Eq. (14)):

$$
\frac{\partial M_{B}}{\partial z_{1}}+\frac{\partial M_{B}}{\partial z_{2}}+\frac{\partial M_{B}}{\partial z_{4}}=-2 \lambda z_{1}
$$

with $\lambda$ defined in Eq. (13). The solution for $M_{B}$ is

$$
M_{B}=M_{B}^{B o r n} \Phi_{B}\left(z_{1}-z_{4}, z_{2}-z_{4}\right) e^{-\lambda \ln ^{2} z_{1}} .
$$

If we assume the hierarchy $s>Q^{2}>u$, we can fix the unknown function $\Phi_{B}$ with the matching condition:

$$
M_{B}=\widetilde{M_{B}} \quad \text { for } z_{4}=0,
$$


where $\widetilde{M_{B}}$ is the DVCS amplitude for the backward scattering of a collinear incoming quark and outgoing gluon. It satisfies

$$
\frac{\partial \widetilde{M_{B}}}{\partial z_{1}}+\frac{\partial \widetilde{M_{B}}}{\partial z_{2}}=-2 \lambda z_{1} \widetilde{M_{B}}
$$

which yields the solution

$$
\widetilde{M_{B}}=\widetilde{\Phi}_{B}\left(z_{1}-z_{2}\right) e^{-\lambda \ln ^{2} z_{1}}
$$

Finally, we can specify $\widetilde{\Phi}_{B}$. When $z_{2}=0\left(Q^{2}=\mu^{2}\right), \widetilde{M_{B}}$ must coincide with the amplitude $C_{B}$ for the backward Compton scattering. $C_{B}$ was calculated in the LLA by direct calculation of the relevant Feynman graphs. [16] The IREE for $C_{B}$ is:

$$
\frac{\partial C_{B}}{\partial z_{1}}=-2 \lambda z_{1} C_{B}
$$

with the boundary condition

$$
C_{B}=C_{B}^{B o r n} \quad \text { for } z_{1}=0 \quad\left(s=\mu^{2}\right) .
$$

Combining the above results, we obtain at the final expression:

$$
M_{B}=M_{B}^{B o r n} e^{-\frac{\alpha_{s}}{4 \pi} C_{F} \ln ^{2}\left(s / Q^{2}\right)} .
$$

\section{Non-forward structure functions}

The DVCS structure functions are a generalization of the conventional DIS structure functions. The novel feature DVCS structure functions is the explicit dependence on the fraction $\zeta$ of the longitudinal momentum $r$ transferred to a hadron, $r=\zeta p$. The $Q^{2}$ evolution of these structure functions is treated in the LLA in the framework of a DGLAP type of equation[2]. Here we consider the non-forward quark structure function in the DLA, which we have applied above to the forward and backward DVCS scattering amplitudes.

We can factorize the amplitude of the DVCS process and express the quark structure function as a four-quark amplitude $M_{i j, s p}(p, k, k-r),(c f$., Fig.5). The two lower quarks in the amplitude $M_{i j, s p}$ carrying the momenta $p$ and $p-r$ are on the mass shell, while two upper ones with the momenta $k$ 
and $k-r$ are virtual. The spinor indices $\{i, j\}$ belong to the lower lines, and they are summed with the indices of the initial and final quark spinors $U_{j}(p)$ and $\bar{U}_{i}(p-r)$. The total amplitude is given by the integral of the function:

$$
\bar{M}_{s p}(p, k, k-r)=\bar{U}_{i}(p-r) M_{i j, s p}(p, k, k-r) U_{j}(p)
$$

with the elementary quark-photon scattering amplitude:

$$
\begin{aligned}
T^{\mu \nu} & =-\int \frac{d^{4} k}{(2 \pi)^{4}} \operatorname{Tr} \bar{M}(p, k, k-r) \times \\
& \times\left[\frac{\hat{k}}{k^{2}} \gamma^{\mu} \frac{\hat{k}+\hat{q}}{(k+q)^{2}} \gamma^{\nu} \frac{\hat{k}-\hat{r}}{(k-r)^{2}}+\frac{\hat{k}}{k^{2}} \gamma^{\nu} \frac{\hat{k}-\hat{q}^{\prime}}{\left(k-q^{\prime}\right)^{2}} \gamma^{\mu} \frac{\hat{k}-\hat{r}}{(k-r)^{2}}\right],
\end{aligned}
$$

The trace in $T^{\mu \nu}$ is taken over the upper line indices $\{s, p\}$.
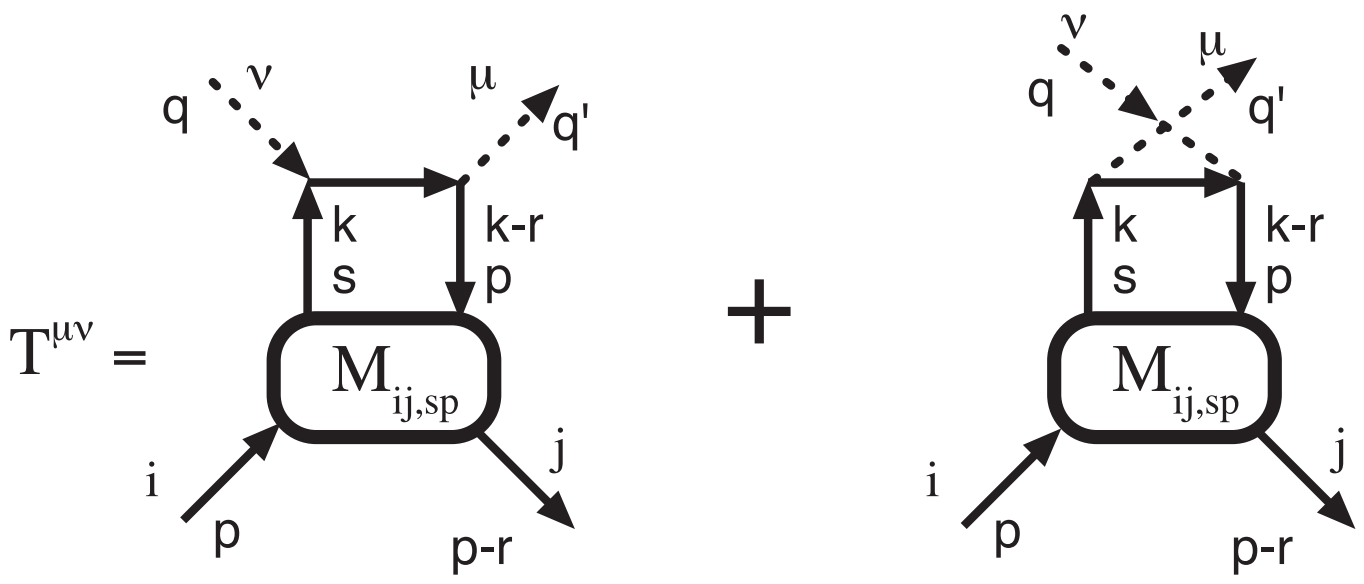

Figure 5: The graphs for the non-forward structure functions

To derive the IREE for the amplitude $M_{i j, s p}(p, k, k-r)$, we decompose this using a complete set of 16 gamma matrices:

$$
M_{i j, s p}=\sum_{A, B=1}^{16} \gamma_{s i}^{A} \gamma_{j p}^{B} M_{A B} .
$$


where the sum runs over the basis set $\gamma^{i}=\left\{1, \gamma^{\lambda}, \sigma^{\lambda \sigma}, \gamma^{\lambda} \gamma^{5}, \gamma^{5}\right\}$.

In the double logarithmic approximation (DLA), the scalar function $M_{A B}$ is comprised of a product of the Born terms which can depend on the invariant energy $s=(k-p)^{2}$ and the virtualities $l_{1}^{2}=k^{2}, l_{2}^{2}=(k-r)^{2}$. Note the $u=(k+p-r)^{2}$ invariant is not independent for $t \approx 0$ since $s+u=l_{1}^{2}+l_{2}^{2}$.

The essential contribution of the amplitude $M_{i j, s p}$ comes only in the region where both virtualities $\left\{l_{1}^{2}, l_{2}^{2}\right\}$ are negative and of the same order, i.e., $l_{1}^{2} \sim$ $l_{2}^{2} \sim l^{2}=-l_{\perp}^{2}$. Therefore, it is natural to write $M_{i j, s p}$ as a sum of an s-channel and a u-channel piece:

$$
\begin{aligned}
M_{i j, s p} & =\frac{1}{s} \sum_{A, B}\left(\gamma^{A}\right)_{s i}\left(\gamma^{B}\right)_{j p} F_{A B}^{S}\left(\frac{s}{\mu^{2}}, \frac{l^{2}}{\mu^{2}}\right) \\
& +\frac{1}{u} \sum_{A, B}\left(\gamma^{A}\right)_{p i}\left(\gamma^{B}\right)_{j s} F_{A B}^{U}\left(\frac{-u}{\mu^{2}}, \frac{l^{2}}{\mu^{2}}\right)
\end{aligned}
$$

It is easy to verify that there is no double logarithmic (DL) contribution to the IREE in the kinematic region $l^{2} \neq 0$ since $l_{\perp}^{2}$ plays the role of the infrared cut-off (instead of $\mu^{2}$ ). Thus,

$$
\begin{aligned}
& \left(s \frac{\partial}{\partial s}+\frac{\partial}{\partial z}\right) F_{A B}^{S}=0 \\
& \left(u \frac{\partial}{\partial u}+\frac{\partial}{\partial z}\right) F_{A B}^{U}=0
\end{aligned}
$$

where we define

$$
z=\ln \frac{l^{2}}{\mu^{2}}
$$

In terms of a Mellin transform,

$$
F_{A B}^{S}=\int \frac{d \omega}{2 \pi i}\left(\frac{s}{\mu^{2}}\right)^{\omega} f_{A B}^{S}(\omega, z)
$$

with a corresponding definition for $u$-channel function $F_{A B}^{U}$. These functions satisfy the differential equation:

$$
\frac{\partial}{\partial z} f_{A B}^{S, U}=-\omega f_{A B}^{S, U}
$$


We can write the solution as:

$$
f_{A B}^{S, U}(\omega, z)=R_{A B}^{S, U}(\omega) e^{-\omega z},
$$

where the function $R_{A B}^{S, U}$ is determined by the boundary conditions in the limit where virtualities become vanishingly small, $l^{2} \sim \mu^{2}$ which implies $z=0$. This kinematic region is the Regge limit which describes the scattering of on-shell quarks with approximately zero momentum transfer.

The symbol $R_{A B}^{S, U}$ is the coefficient of the Regge amplitude when expanded in the $\left\{\gamma^{A}, \gamma^{B}\right\}$ basis. We can introduce an amplitude $A_{\text {Regge }}^{h, h^{\prime}, f f^{\prime}}$ which corresponds to the amplitude $M_{i j, s p}$ in the Regge limit, and expand this in the $\left\{\gamma^{A}, \gamma^{B}\right\}$ basis as follows:

$$
\begin{aligned}
A_{\text {Regge }}^{h, h^{\prime}, f, f^{\prime}} & =\sum_{i j s p} \bar{U}_{s}^{h}\left(q^{\prime}\right) U_{i}^{h^{\prime}}(p) T_{i j, s p}^{\text {Regge }} \bar{U}_{j}^{f^{\prime}}(p) U_{p}^{f}\left(q^{\prime}\right) \\
& =\frac{1}{s} \sum_{A, B} \bar{U}^{h}\left(q^{\prime}\right) \gamma^{A} U^{h^{\prime}}(p) \cdot \bar{U}^{f^{\prime}}(p) \gamma^{B} U^{f}\left(q^{\prime}\right) R_{A B}^{S} \\
& +\frac{1}{u} \sum_{A, B} \bar{U}^{f}\left(q^{\prime}\right) \gamma^{A} U^{h^{\prime}}(p) \cdot \bar{U}^{f^{\prime}}(p) \gamma^{B} U^{h}\left(q^{\prime}\right) R_{A B}^{U},
\end{aligned}
$$

where $h, f, h^{\prime}$, and $f^{\prime}$ are the quark helicities.

The spinor structure of the Regge amplitude is determined by the Born terms, so we have

$$
T_{i j, s p}^{\text {Regge }}=\frac{1}{s} \gamma_{s i}^{\lambda} \gamma_{j p}^{\sigma} g^{\lambda \sigma} R_{S}+\frac{1}{u} \gamma_{p i}^{\lambda} \gamma_{j s}^{\sigma} R_{U}
$$

In the Regge theory framework, it is more natural to work with amplitudes of definite signature rather than s-channel and u-channel amplitudes, rather than with the forward and backward ones, therefore, we work with $R^{ \pm}$defined by:

$$
\begin{aligned}
& R_{S}=R^{(+)}+R^{(-)} \\
& R_{U}=R^{(+)}-R^{(-)} .
\end{aligned}
$$

The form of the Regge amplitude dictates the final structure for the fourquark amplitude:

$$
\begin{aligned}
M_{i j, s p}\left(s, l^{2}\right) & =\frac{1}{s}\left(\gamma^{\lambda}\right)_{s i}\left(\gamma^{\sigma}\right)_{j p} g^{\lambda \sigma} \int \frac{d \omega}{2 \pi i} R_{S}(\omega)\left(\frac{s}{l^{2}}\right)^{\omega} \\
& +\frac{1}{u}\left(\gamma^{\lambda}\right)_{p i}\left(\gamma^{\sigma}\right)_{j s} g^{\lambda \sigma} \int \frac{d \omega}{2 \pi i} R_{U}(\omega)\left(\frac{s}{l^{2}}\right)^{\omega}
\end{aligned}
$$


To extract the non-forward structure function, the four-quark amplitude must be substituted into the total amplitude. For $t \approx 0$, the total amplitude is given by the sum of the two transverse tensors,

$$
T^{\mu \nu}=\frac{1}{2 p \cdot q^{\prime}} g_{\perp}^{\mu \nu} T_{1}-\frac{1}{2 p \cdot q^{\prime}} i \varepsilon^{\mu \nu \rho \eta} \frac{1}{p \cdot q^{\prime}} T_{2},
$$

where $T_{1}$ is symmetric, and $T_{2}$ is antisymmetric. We can express $T_{1}$ and $T_{2}$ in terms of the quark $(q)$ and antiquark $(\bar{q})$ non-forward structure functions $\mathcal{F}_{\zeta}^{q, \bar{q}}(x)$ and $G_{\zeta}^{q, \bar{q}}(x)$,

$$
\begin{aligned}
& T_{1}=\bar{U}(p-r) \hat{q}^{\prime} U(p) \int_{0}^{1} d x\left[\frac{1}{x-\zeta+i \delta}+\frac{1}{x-i \delta}\right]\left(\mathcal{F}_{\zeta}^{q}(x)+\mathcal{F}_{\zeta}^{\bar{q}}(x)\right), \\
& T_{2}=\bar{U}(p-r) \hat{q}^{\prime} \gamma^{5} U(p) \int_{0}^{1} d x\left[\frac{1}{x-\zeta+i \delta}-\frac{1}{x-i \delta}\right]\left(G_{\zeta}^{q}(x)+G_{\zeta}^{\bar{q}}(x)(48)\right.
\end{aligned}
$$

(Here we follow the definitions given in Refs. [2].)

Introducing the Sudakov variables, the explicit expressions for $T_{1,2}$ are:

$$
\begin{aligned}
T_{1} & =\int \frac{d^{4} k}{(2 \pi)^{4} i} \operatorname{Tr} \bar{M}(p, k, k-r) \hat{k} \hat{q}^{\prime}(\hat{k}-\hat{r}) \frac{1}{k^{2}(k-r)^{2}} \\
& \times\left[\frac{1}{\beta-\zeta+i \delta}+\frac{1}{\beta-i \delta}\right], \\
T_{2} & =-\int \frac{d^{4} k}{(2 \pi)^{4} i} \operatorname{Tr} \bar{M}(p, k, k-r) \hat{k} \hat{q}^{\prime} \gamma^{5}(\hat{k}-\hat{r}) \frac{1}{k^{2}(k-r)^{2}} \\
& \times\left[\frac{1}{\beta-\zeta+i \delta}-\frac{1}{\beta-i \delta}\right],
\end{aligned}
$$

with

$$
\begin{aligned}
d^{4} k & =\frac{s^{\prime}}{2} d \alpha d \beta d k_{\perp}, \\
s^{\prime} & =2 p \cdot q^{\prime} .
\end{aligned}
$$

The integrals over $\beta$ are restricted to the interval $0 \leq \beta \leq 1$ which follows from the position of the poles in the $\alpha$-plane. Examining these expressions demonstrate that to find the structure functions, the amplitude $\bar{M}$ must be integrated over the virtual momentum $k$ while keeping $\beta$ fixed. In performing 
this integration, we need only keep the terms of the form $d \alpha / \alpha d k_{\perp}^{2} / k_{\perp}^{2}$ for DL accuracy. These terms come from the region where

$$
\begin{aligned}
k^{2} & =\alpha \beta s^{\prime}-k_{\perp}^{2} \approx-k_{\perp}^{2} \\
(k-r)^{2} & =\alpha(\beta-\zeta) s^{\prime}-k_{\perp}^{2} \approx-k_{\perp}^{2} \\
s=(k-p)^{2} & =\alpha(\beta-1) s^{\prime}-k_{\perp}^{2} \approx \alpha(\beta-1) s^{\prime} \\
u=(k+p-r)^{2} & =\alpha(\beta+1-\zeta) s^{\prime}-k_{\perp}^{2} \approx \alpha(\beta+1-\zeta) s^{\prime} .
\end{aligned}
$$

These relations simplify the process of extracting the Regge limit. First, they imply that both virtualities $\left\{l_{1}^{2}, l_{2}^{2}\right\}$ in the four-quark amplitude must be of the same order, i.e., $l_{1}^{2} \sim l_{2}^{2} \sim-k_{\perp}^{2}$; this justifies the assumption that was adopted previously. Second, only the quadratic terms in $k_{\perp}$ from the spinor trace are necessary for the non-forward amplitudes $T_{1,2}$. We can then compute these traces as follows:

$$
\begin{aligned}
\operatorname{Tr} \bar{M} \hat{k}_{\perp} \hat{q}^{\prime} \hat{k}_{\perp} & =\bar{U}(p-r) \gamma^{A} \hat{k}_{\perp} \hat{q}^{\prime} \hat{k}_{\perp} \gamma^{B} U(p)\left(\frac{1}{s} F_{A B}^{S}+\frac{1}{u} F_{A B}^{U}\right) \\
& =-2\left(\frac{1}{s} R_{S}+\frac{1}{u} R_{U}\right) k_{\perp}^{2} \bar{U}(p-r) \hat{q}^{\prime} U(p) \\
\operatorname{Tr} \bar{M} \hat{k}_{\perp} \hat{q}^{\prime} \gamma^{5} \hat{k}_{\perp} & =\bar{U}(p-r) \gamma^{A} \hat{k}_{\perp} \hat{q}^{\prime} \gamma^{5} \hat{k}_{\perp} \gamma^{B} U(p)\left(\frac{1}{s} F_{A B}^{S}+\frac{1}{u} F_{A B}^{U}\right) \\
& =2\left(\frac{1}{s} R_{S}+\frac{1}{u} R_{U}\right) k_{\perp}^{2} \bar{U}(p-r) \hat{q}^{\prime} \gamma^{5} U(p) .
\end{aligned}
$$

To calculate the structure functions, we must replace the infrared cut-off $\mu^{2}$ in the Regge amplitude by $-k_{\perp}^{2}$, and then integrate over the loop momentum. The forward and backward amplitudes will yield the quark and antiquark functions, respectively. In the DL region, the transverse momentum is

$$
k_{\perp}^{2} \geq \operatorname{Max}\left\{|\alpha \beta| s^{\prime},|\alpha(\beta-\zeta)| s^{\prime}\right\} \quad .
$$

The remaining integral over $\alpha$ is purely logarithmic, and the symmetric integration limit yield $i \pi$.

Finally, working with DL accuracy we retain only the non-vanishing terms in the limit $\omega \rightarrow 0$ to arrive at the following structure functions:

$$
\begin{aligned}
\mathcal{F}_{\zeta}^{q}(\beta) & =\frac{1}{8 \pi^{2}} C_{F} \frac{1}{1-\beta} \int \frac{d \omega}{2 \pi} R_{S}(\omega) \frac{1}{\omega} \times \\
& \times\left[\theta\left(\beta-\frac{1}{2} \zeta\right)\left(\frac{1-\beta}{\beta}\right)^{\omega}+\theta\left(\frac{1}{2} \zeta-\beta\right)\left(\frac{1-\beta}{\zeta-\beta}\right)^{\omega}\right]
\end{aligned}
$$




$$
\begin{aligned}
\mathcal{F}_{\zeta}^{\bar{q}}(\beta) & =\frac{1}{8 \pi^{2}} C_{F} \frac{1}{1+\beta-\zeta} \int \frac{d \omega}{2 \pi} R_{U}(\omega) \frac{1}{\omega} \times \\
& \times\left[\theta\left(\beta-\frac{1}{2} \zeta\right)\left(\frac{1+\beta-\zeta}{\beta}\right)^{\omega}+\theta\left(\frac{1}{2} \zeta-\beta\right)\left(\frac{1+\beta-\zeta}{\zeta-\beta}\right)^{\omega}\right]
\end{aligned}
$$

The explicit expressions for $R_{S}, R_{U}$ can be taken from Refs. [11, 9]. The functions $G_{\zeta}^{q, \bar{q}}(\beta)$ are expressed by Regge amplitudes using the same formula. The integral over $\omega$ runs along imaginary axes to the right of the singularities of the $R_{S, U}$ functions. Non-zero contributions exist only when the arguments in the brackets are larger than unity, so that the actual support of the first term on the RHS of Eq. (50) and Eq. (51) is $\zeta / 2 \leq \beta \leq 1 / 2$. The Regge limit implies the argument of the Mellin transform is be asymptotically large, which corresponds to the the region where $\zeta \ll 1$ and $\beta \sim \zeta$.

The absence of a $Q^{2}$ dependence in these expressions resembles the DGLAP type of evolution equation for the non-forward structure functions in $Q^{2} \rightarrow \infty$ limit. In contrast to the DGLAP evolution equations, the DVCS evolution equations are not restricted to the region $\beta \leq \zeta$.

Notice that for $\beta=0$, we find that $\mathcal{F}_{\zeta}^{q, \bar{q}}(0) \neq 0$ in contrast to the result for the DGLAP non-forward functions. The Mellin moments $M_{n}^{q, \hat{q}}(\zeta)=$ $\int_{0}^{1} d x x^{n-1} \mathcal{F}_{\zeta}^{q, \hat{q}}(x)$ for the integer $n$ are not necessarily polynomials of degree $n$ with respect to the transfer momentum fraction $\zeta$, as in the DGLAP case.

\section{Conclusion}

We obtained the explicit expressions for the DVCS amplitudes in the Regge limits of forward and backward scattering. For the forward scattering region, the asymptotic amplitude takes the form of the DIS structure functions times a Sudakov exponential factor of the form $\exp \left[-\left(\alpha_{s} / 4 \pi\right) C_{F} \ln ^{2}\left(-t / \mu^{2}\right)\right]$. The DVCS amplitude in the backward scattering region is purely of the Sudakov type with an exponential factor of the form $\exp \left[-\left(\alpha_{s} / 4 \pi\right) C_{F} \ln ^{2}\left(s / \mu^{2}\right)\right]$. We have not used the DGLAP evolution equations which assume the transverse momenta to be strictly ordered in virtuality. This enables us to take into account important logarithmic contributions that are beyond of the realm of the DGLAP approach. Specifically, there is no ordering of the transverse momenta in the Regge region, and this allows the internal transverse momenta to exceed $Q^{2}$, in contrast to the DGLAP method. Such contributions lead to the power-like behavior of the DVCS amplitude. 


\section{Acknowledgments}

This work is supported in part by Grant INTAS-RFBR-95-311, Grant RFBR98-02-17629, the US Department of Energy, and the Lightner-Sams Foundation. BIE thanks the SMU Theory Group for their kind hospitality and the Lightner-Sams Foundation for financial support during the period in which part of this research was carried out.

\section{References}

[1] X.Ji, Phys.Rev.Lett. 78 (1997) 610; Phys.Rev.D55 (1997) 7114

[2] A.V.Radyushkin, Phys.Lett. B380 (1996) 417, B385 (1996) 333; Phys.Rev. D56 (1997) 5524.

[3] S.J.Brodsky, L.Frankfurt, J.F.Gunioni, A.H.Mueller and M.Strikman, Phys.Rev. D50 (1994) 3134. J.C.Collins, L.Frankfurt and M.Strikman, Phys.Rev. D56 (1997) 2982. Z.Chen, Nucl.Phys.B525 (1998) 369.

[4] G.Altarelli and G.Parisi, Nucl.Phys B126 (1977) 297 V.N.Gribov and L.N.Lipatov, Sov.J.Nucl.Phys 15，438， 675 (1972) Yu.L.Dokshitser, Sov.Phys.JETP 46 (1977) 641

[5] X.Ji, G.Nucl.Part.Phys.24 (1998) 1181. Also references therein.

[6] G.Altarelli, R.D.Ball, S.Forte and G.Ridolfi, Nucl.Phys. B496 (1997) 337.

[7] J.Blumlein and A.Vogt, Phys. Lett. B370 (1996) 149; Acta Phys. Pol. B27 (1996) 1309; Phys. Lett. B386 (1996) 350.

[8] J.Blumlein, B.Geyer and D.Robaschik, Phys. Lett. B406 (1997) 161; hep-ph/9711405.

[9] V.S.Fadin, E.A.Kuraev and L.N.Lipatov, Sov.Phys.JETP 44 (1976) 443, ibid 45 (1977) 199. Y.Y.Balitskij and L.N.Lipatov, Sov.J.Nucl.Phys.28 (1978) 822 . 
[10] B.I.Ermolaev, S.I.Manayenkov and M.G.Ryskin, Z.Phys. C69 (1996) 259; J.Bartels, B.I.Ermolaev and M.G.Ryskin, Z.Phys. C70 (1996) 273; Z.Phys. C72 (1996) 627.

[11] R.Kirschner and L.N.Lipatov, Nucl.Phys. B213 (1983) 122

[12] B.I.Ermolaev and L.N.Lipatov, Int.J.Mod.Phys. A4 (1989) 3147

[13] V.N.Gribov, Yad.Fiz. 5 (1967) 399

[14] B.I.Ermolaev, V.S.Fadin and L.N.Lipatov, Yad.Fiz. 45 (1987) 817

[15] M.Chaichian and B.Ermolaev, Nucl.Phys. B451 (1995) 194

[16] V.G.Gorshkov, V.N.Gribov and G.V.Frolov. Sov.Phys. JETP 51 (1966) 1093.

[17] B.I.Ermolaev and M.Krawczyk, Preprint IFT- 20- 91. 